\title{
Clinical and Radiological Results of Hook Plate Fixation in Acute Acromioclavicular Joint Dislocations and Distal Clavicle Fractures
}

\author{
Joo Han Oh, Seunggi Min", Jae Wook Jung, Hee-June Kim, Jae Yoon Kim², Seok Won Chung ${ }^{3}$, \\ Joon Yub Kim ${ }^{4}$, Jong Pil Yoon ${ }^{1 凶}$
}

Department of Orthopaedic Surgery, Seoul National University Bundang Hospital, Seoul National University College of Medicine, Seongnam, ${ }^{1}$ Department of Orthopaedic Surgery, Kyungpook National University, School of Medicine, Daegu, ${ }^{2}$ Department of Orthopaedic Surgery, Chung-Ang University School of Medicine, ${ }^{3}$ Department of Orthopaedic Surgery, Konkuk University School of Medicine, Seoul, ${ }^{4}$ Department of Orthopaedic Surgery, Myongji Hospital, Goyang, Korea

Background: The purpose of this study was to evaluate the clinical outcomes and complications of hook plate fixation in acromioclavicular (AC) joint dislocations and distal clavicle fractures.

Methods: We retrospectively reviewed a series of 60 consecutive patients with hook plate fixation for AC joint dislocation (group I) and distal clavicle fracture (group II). Groups I and II had 39 and 21 patients, respectively. Clinical results were evaluated using the pain visual analogue scale (VAS), simple shoulder test, and Constant-Murley scores. In addition, subacromial erosion and stiffness were evaluated as complications.

Results: At the removal, the pain VAS was $2.69 \pm 1.30$ and $4.10 \pm 2.14$ in groups I and II, respectively, which were significantly different $(p=0.003)$. The simple shoulder test score was $9.59 \pm 1.60$ and $7.81 \pm 2.67$ in groups I and II, respectively, which were also significantly different $(p=0.002)$. Subacromial erosion was significantly more frequent in group II $(14 / 21$ patients, $66.7 \%)$ than in group I $(15 / 39$ patients, 38.5\%) $(p=0.037)$, and stiffness was also higher in group II $(17 / 21$ patients, $81.0 \%)$ than in group I (22/39 patients, $56.4 \%)$, but it was not significant.

Conclusions: Hook plate fixation showed good clinical and functional results for the treatment of acute unstable AC joint dislocation and distal clavicle fracture. But, in distal clavicle fractures, there are more subacromial erosion and stiffness compare with acute unstable AC joint dislocation.

(Clin Shoulder Elbow 2018;21(2):95-100)

Key Words: Joint dislocation; Clavicle; Fracture; Shoulder

\section{Introduction}

Acromioclavicular (AC) joint dislocation is a common injury that accounts for about $50 \%$ of shoulder injuries from sports. ${ }^{1,2)}$ AC joint dislocation is classified according to the Rockwood classification based on the severity. Controversy exists in this classification, but only dislocations of type III or higher have surgical indications. Surgery is performed using a K-wire, coracoclavicular (CC) screw, tight loop, and hook plate. ${ }^{3)}$
In addition, distal clavicle fracture accounts for $15 \%$ to $25 \%$ of all clavicle fractures, and the treatment depends on the classification of the fracture. ${ }^{4,5)}$ If a surgical treatment is considered, instruments such as K-wire, tension band wire, and various types of plate can be used. ${ }^{6)}$

Among the various surgical procedures, hook plate is a device that can be used in both AC joint dislocation and distal clavicle fracture. It is easy and convenient to operate, and it has the advantage of showing stiffness similar to that of the original $\mathrm{AC}$ joint

Received January 31, 2018. Revised March 16, 2018. Accepted March 18, 2018.

Correspondence to: Jong Pil Yoon

Department of Orthopedic Surgery, Kyungpook National University, School of Medicine, 130 Dongdeok-ro, Jung-gu, Daegu 41944, Korea

Tel: +82-53-420-5628, Fax: +82-53-422-6605, E-mail: jpyoon@knu.ac.kr, ORCID: https://orcid.org/0000-0001-6446-6254

IRB approval: Kyungpook National University Hospital (No. KNUH 2012-09-042).

Financial support: This research was supported by the Basic Science Research Program through the National Research Foundation of Korea, funded by the Ministry of Science, ICT and Future Planning (2015R1C1A1A02036478). Conflict of interests: None. 
due to its rigid fixation. ${ }^{7,8)}$ Therefore, it is possible to reduce the patient's immobilization period and to promote early rehabilitation, resulting in rapid healing of conoid and trapezoid ligaments as reported by previous studies. ${ }^{9,10)}$

However, despite the advantages described above, the hook plate has several problems. The hook plate may induce a pressure rise of the hook under the surface of the acromion, which causes erosion. ${ }^{11,12)}$ Moreover, because the plate design allows the normal biomechanics of the AC joint to be maintained, the hook moves freely as the clavicle rotates and elevates when the arm is elevated. ${ }^{13)}$ Therefore, the hook plate may cause impingement, osteolysis, arthritis of the AC joint, and other complications, such as calcification and ossification of the CC ligament. ${ }^{1,14)}$ Although there are many reports of $\mathrm{AC}$ joint dislocation and distal clavicle fractures treated with a hook plate, there have been few reports comparing AC joint dislocation with distal clavicle fracture. In addition, there were no studies comparing the incidence of complications. The purpose of this study was 1) to evaluate the clinical and radiological outcomes of hook plate fixation in $\mathrm{AC}$ joint dislocation and distal clavicle fracture and 2) to compare the clinical result and their complications.

\section{Methods}

We retrospectively reviewed a consecutive series of 73 patients with a hook plate for AC joint dislocation and distal clavicle fracture between 2009 and 2015.

For AC joint dislocation, the inclusion criteria included Rockwood lesion types III to $\mathrm{V}$ and a minimum clinical follow-up of 12 months. For distal clavicle fracture, the inclusion criterion is a minimum clinical follow-up of 12 months. We excluded patients with chronic AC joint dislocations, a history of stiff shoulder, AC joint arthritis, and had further surgical intervention or injury to the shoulder girdle. All the study protocols were approved by Institutional Review Board of the Kyungpook National University Hospital (No. KNUH 2012-09-042).

Overall, a total of 60 patients (51 men and 9 women) with an average age of $39.93 \pm 14.45$ years were included in the study. Groups I (AC joint dislocation) and II (distal clavicle fracture) had 39 and 21 patients, respectively. In group 1, there were 15 patients of Rockwood lesion types III, 2 patients of Rockwood lesion types IV, and 22 patients of Rockwood lesion types V. In group 2, there were 15 patients of Neer types II fracture, and 6 patients of Neer types III fracture. All clinical data were prospectively collected in our database and retrospectively reviewed.

All surgeries were performed in both groups in the beach chair position. An incision was made over the clavicle, and the trapezius was opened to expose the AC joint and distal clavicle. In group I, manual reduction of the AC joint dislocation was performed, and the adequate 3.5-mm LCP Clavicle Hook Plate (Synthes Inc., West Chester, PA, USA) was inserted under the surface of the acromion and the screw was fixed to the distal clavicle. In group II, the same approach was used to expose the fracture site of the distal clavicle and to perform a reduction of fracture fragment first. If needed, after fixing with a tension band wire for interfragmentary fixation, the hook plate was applied. Fourteenpatients had additional tension band wiring. Additional screws or wiring was used if necessary. In both groups, the trapezius was tightly sutured after hook plate fixation using the same technique.

After 2 weeks of operation, all patients were allowed to use their arm for daily activities, and rehabilitation was progressively initiated. After 5 weeks, the patients were allowed to move freely. Resumption of strength-related activities was permitted 3 months postoperatively. Removal of the hook plate was performed after 4 to 6 months in group I, whereas in group II, the hook plate was removed after confirming bone union. As a result, there was a significant difference in the period from operation to removal in both groups (group I: $5.31 \pm 2.23$ months, group II: $9.65 \pm 1.78$ months, $p=0.001$ ). Subacromal erosion was evaluated using simple radiograph as previous studies described. ${ }^{11,15)}$

All patients were followed-up at least 1 year after the operation. The clinical outcome assessment was evaluated at the removal time and at the final follow-up using the visual analogue scale (VAS), simple shoulder test, and Constant-Murley scores by an independent researcher who was not involved in the patient treatment. The final clinical assessment was performed at 24.90 \pm 5.43 months and $26.01 \pm 4.19$ months in groups I and II, respectively. Shoulder stiffness for forward elevation was defined as $120^{\circ}$ passively; external rotation, as $30^{\circ}$ passively; and internal rotation at the back, as lower than $\mathrm{L} 3$ passively. ${ }^{16)}$ A clinical

Table 1. Preoperative and Baseline Data

\begin{tabular}{|cccc}
\hline \multicolumn{1}{c}{ Variable } & Group I & Group II & $p$-value \\
\hline No. of patient & 39 & 21 & \\
\hline Age (yr) & $40.10 \pm 15.04$ & $39.62 \pm 13.65$ & 0.903 \\
\hline Sex (male:female) & $35: 4$ & $16: 5$ & 0.161 \\
\hline $\begin{array}{l}\text { Side of involvement } \\
\text { (dominant:non-dominant) }\end{array}$ & $13: 8$ & $23: 16$ & 0.825 \\
\hline $\begin{array}{l}\text { Time interval (from injury to } \\
\text { surgery) (d) }\end{array}$ & $9.51 \pm 8.41$ & $7.95 \pm 7.79$ & 0.485 \\
\hline $\begin{array}{l}\text { Etiology } \\
\text { Fall down }\end{array}$ & 4 & & 0.236 \\
\hline Slip down & 14 & 4 & \\
\hline Traffic accident & 3 & 22 & NA \\
\hline Rockwood classification & III: $13, \mathrm{~V}: 26$ & - & \\
\hline
\end{tabular}

Values are presented as number only or mean \pm standard deviation. Group I: patients with hook plate fixation for acromioclavicular joint dislocation, Group II: patients with distal clavicle fracture, NA: not available. 
researcher measured the range of motion of each patient with a goniometer.

Radiologic assessment of reduction and reduction-loss of AC joint dislocation was performed preoperatively, postoperatively, and at the final follow-up using plain radiographs, which were compared with the normal side. The images were analyzed and standardized to assess the coracoclavicular distance $(C C D$, height [in percent] to the contralateral shoulder between the upper border of the coracoid process and the inferior cortex of the clavicle). Reduction loss after plate removal was defined as mild (CCD $<50 \%$ comparing with normal side), severe (CCD 50\%-100\%), and re-dislocation (CCD $>100 \%)^{.8)}$

Statistical analysis was performed using SPSS Statistics ver. 17.0 (SPSS Inc., Chicago, IL, USA), and the null hypotheses were rejected when $p$-values were $<0.05$. Descriptive statistics, Student's t-test, and Mann-Whitney U-test were used to determine the outcome differences between the two treatment groups.

\section{Results}

There was no significant baseline difference between both groups in terms of age, side of involvement (dominant or nondominant), mechanism of injury, and the time from injury to surgery (Table 1).

In the clinical results at the removal time, the VAS was 2.69 \pm 1.30 and $4.10 \pm 2.14$ in groups I and II, respectively, which showed significant differences $(p=0.003)$ (Table 2$)$. The simple shoulder test score was $9.59 \pm 1.60$ and $7.81 \pm 2.67$ in groups I and II, respectively, which also showed significant differences $(p=0.002)$. The Constant-Murley score was $86.18 \pm 7.25$ and $84.29 \pm 7.53$ in groups I and II, respectively, which showed a significant difference $(p=0.345)$ (Table 2$)$.

At the final follow-up, mean VAS $(1.15 \pm 0.98$ vs. $1.43 \pm$ $1.07)$, simple shoulder test $(11.03 \pm 1.11$ vs. $10.71 \pm 1.23)$, and Constant-Murley scores (94.49 \pm 5.38 vs. $92.14 \pm 5.57)$

Table 2. Clinical Results of the Two Groups

\begin{tabular}{|c|c|c|c|}
\hline Variable & Group I & Group II & $p$-value \\
\hline No. of patient & 39 & 21 & \\
\hline Removal time (d) & $159.31 \pm 66.98$ & $289.38 \pm 53.52$ & $0.001^{*}$ \\
\hline Postoperative VAS at removal & $2.69 \pm 1.30$ & $4.10 \pm 2.14$ & $0.003^{*}$ \\
\hline Postoperative simple shoulder test score at removal & $9.59 \pm 1.60$ & $7.81 \pm 2.67$ & $0.002^{*}$ \\
\hline Postoperative Constant-Murley score at removal & $86.18 \pm 7.25$ & $84.29 \pm 7.53$ & 0.345 \\
\hline Stiffness at removal (yes:no) & $22: 17$ & $17: 4$ & 0.057 \\
\hline Subacromial erosion at removal (yes:no) & $15: 24$ & $14: 7$ & $0.037^{\star}$ \\
\hline Final follow-up (mo) & $24.90 \pm 5.43$ & $26.01 \pm 4.19$ & 0.418 \\
\hline Postoperative VAS at final follow-up & $1.15 \pm 0.98$ & $1.43 \pm 1.07$ & 0.323 \\
\hline Postoperative simple shoulder test at final follow-up & $11.03 \pm 1.11$ & $10.71 \pm 1.23$ & 0.340 \\
\hline Postoperative Constant-Murley score at final follow-up & $94.49 \pm 5.38$ & $92.14 \pm 5.57$ & 0.118 \\
\hline Stiffness at final follow-up & $3: 36$ & $2: 19$ & 0.578 \\
\hline
\end{tabular}

Values are presented as number only or mean \pm standard deviation.

Group I: patients with hook plate fixation for acromioclavicular joint dislocation, Group II: patients with distal clavicle fracture, VAS: visual analogue scale. ${ }^{*} p<0.05$.
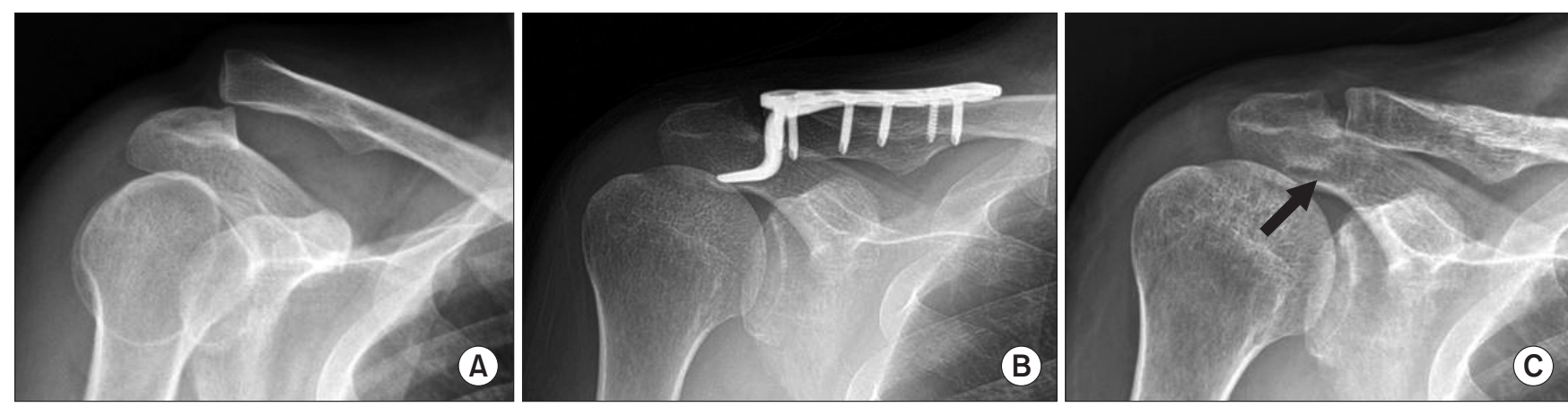

Fig. 1. Serial radiographs of a 36-year-old man treated with a hook plate fixation for acromioclavicular joint dislocation. Preoperative (A) and postoperative 4 months (B) showing good maintenance of joint reduction and subacromial erosion (arrow) after plate removal (C). 

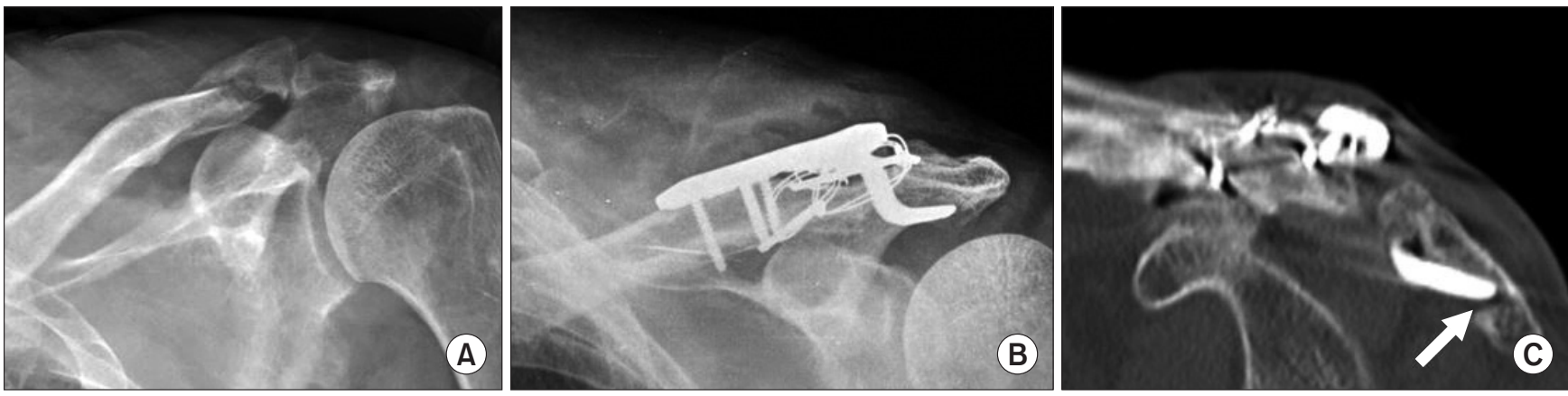

Fig. 2. (A) A 53-year-old man treated with a hook plate fixation for distal clavicle fracture. (B, C) A markedly severe subacromial erosion (arrow) was present eight months postoperatively, which was caused by the hook plate.

were not significant in both groups $(p=0.323,0.340$, and 0.118 , respectively) (Table 2).

At the time of removal, subacromial erosion was significantly more frequent in group II (14/21 patients, $66.7 \%)$ than in group I (15/39 patients, $38.5 \%)(p=0.037)$ (Fig. 1,2$)$, and stiffness was also higher in group II (17/21 patients, $81.0 \%)$ than in group I (22/39 patients, $56.4 \%)$, but not significant $(p=0.057)$. At the final follow-up, patients recovered from stiffness with no significant difference in both groups ( 3 vs. $2, p=0.578$ ) (Table 2).

With regard to the maintenance of the AC joint reduction, there were 3 mild subluxation cases $(C C D<50 \%)$ after plate removal and no re-dislocation in group I. In group II, there was 1 partial nonunion case. However, no other complications requiring surgical revision occurred in either group.

\section{Discussion}

The purpose of this study was to compare the results of $\mathrm{AC}$ joint dislocation and distal clavicle fracture using a hook plate. The overall final clinical results were good in both groups as reported in previous studies. In addition, we aimed to compare the incidence of complications of two other injuries using the same hook plate as well as their favorable good clinical results in our study.

A variety of surgical procedures have been tried to treat $A C$ joint dislocation and distal clavicle fractures before using a hook plate. K-wires, tension band wires, metal screw, and anatomical locking plates have also been used. ${ }^{17-20)}$ However, many complications such as loss of reduction, loosening, osteolysis, and migration of internal fixation frequently occurred in each case. In addition, due to other problems such as adjacent nerve or vascular injury induced by the internal fixation failure internal, ${ }^{15,21)}$ a new surgical method using a hook plate was developed and used until now. Surgical treatment of AC joint dislocation and distal clavicle fracture using a hook plate is relatively easy because of the small incision and minimal dissection. In addition, joint articulation can be preserved, and it is advantageous for early rehabilitation of patients because of its firm fixation. ${ }^{22)}$
Patients with AC joint dislocations and distal clavicle fractures who underwent surgery with a hook plate have been reported to have good surgical results. Di Francesco et al. ${ }^{9)}$ performed an operation using a hook plate in 42 patients with Rockwood type III and V AC joint dislocations. They removed the plate at 1 to 3 months postoperatively, and reported CC ligament healing in 37 patients (88.1\%) on follow-up magnetic resonance imaging at 18 months. Good et al. ${ }^{23)}$ reported a 95\% union rate and no complication in 36 patients with displaced distal clavicle fractures (Neer type II) who underwent surgery with a hook plate. However, there have been no studies comparing both AC joint dislocation and distal clavicle fracture, and both of our groups showed good results in our study.

However, the greatest disadvantage of the hook plate is subacromial erosion and impingement caused by the hook placed under the surface of the acromion. Eschler et al. ${ }^{11)}$ reported that 5 of the 27 cases $(18.5 \%)$ showed signs of subacromial erosion on plain radiographs. Lin et al. ${ }^{15)}$ treated 40 patients with AC joint dislocations or distal clavicle fractures with a hook plate and reported that 15 patients (37.5\%) and 20 patients (50.0\%) showed subacromial impingement syndrome and subacromial erosion, respectively, as revealed by dynamic sonography. They advocated plate removal immediately after obtaining bone union or ligamentous healing. In addition, AC arthritis may occur after the hook plate removal, which may cause pain persistence. As a result, these erosions and other complications cause glenohumeral joint stiffness.

In our study, distal clavicle fractures had more subacromial erosion and stiffness. Erosion was significantly more common in the distal clavicle fracture group than in the AC joint dislocation group ( $p=0.037)$, and the stiffness was more frequent, although with borderline significance $(p=0.057)$.

Therefore, we believe that the following are the possible explanations for these results: First, hook plate removal is performed relatively early in the AC joint dislocation group. In the literature review, clinical and radiological results were good, without complications, in patients whose plates were removed within 3 months from fixation ${ }^{3,9,21,24)}$ compared to that in patients 
with delayed removal. ${ }^{25,26)}$ Second, in distal clavicle fractures, a bone union period is needed, which requires the removal of the hook plate. In most studies, the union of the distal clavicle fracture could last up to 8 months; therefore, it was difficult to remove the hook plate until complete bone healing. ${ }^{27,28)}$

Several limitations should be noted when interpreting our findings. First, our study has limitations that are inherent to those of similar retrospective and small-sized studies. Second, subacromial erosion is difficult to evaluate accurately with radiographs. Therefore, it should be noted that highly precise determination of the presence and severity of erosion can still be elusive. Third, in the distal clavicle fracture, the AC joint was not injured as compared to that on AC joint dislocation. Therefore, the possibility that it may have affected the postoperative clinical outcome cannot be ruled out. Fourth, the bone union of distal clavicle fractures in our study were checked by simple radiograph. Estimation of bone union of distal clavicle fractures is hard by simple radiograph. It may cause incorrect determinations for complete bone union.

\section{Conclusion}

Hook plate fixation showed good clinical and functional results for the treatment of acute unstable AC joint dislocation and distal clavicle fracture. But, in distal clavicle fractures, there are more subacromial erosion and stiffness compare with acute unstable AC joint dislocation.

\section{References}

1. EIMaraghy AW, Devereaux MW, Ravichandiran K, Agur AM. Subacromial morphometric assessment of the clavicle hook plate. Injury. 2010;41(6):613-9.

2. Oussedik S. Injuries to the clavicle and acromioclavicular joint. Br J Hosp Med (Lond). 2007;68(4):M68-70.

3. Tauber M. Management of acute acromioclavicular joint dislocations: current concepts. Arch Orthop Trauma Surg. 2013; 133(7):985-95.

4. Edwards DJ, Kavanagh TG, Flannery MC. Fractures of the distal clavicle: a case for fixation. Injury. 1992;23(1):44-6.

5. van der Meijden OA, Gaskill TR, Millett PJ. Treatment of clavicle fractures: current concepts review. J Shoulder Elbow Surg. 2012;21(3):423-9.

6. Rieser GR, Edwards K, Gould GC, Markert RJ, Goswami T, Rubino LJ. Distal-third clavicle fracture fixation: a biomechanical evaluation of fixation. J Shoulder Elbow Surg. 2013;22(6):84855.

7. McConnell AJ, Yoo DJ, Zdero R, Schemitsch EH, McKee MD. Methods of operative fixation of the acromio-clavicular joint: a biomechanical comparison. J Orthop Trauma. 2007;21(4): 248-53.
8. Yoon JP, Lee BJ, Nam SJ, et al. Comparison of results between hook plate fixation and ligament reconstruction for acute unstable acromioclavicular joint dislocation. Clin Orthop Surg. 2015;7(1):97-103.

9. Di Francesco A, Zoccali C, Colafarina O, Pizzoferrato R, Flamini S. The use of hook plate in type III and $\mathrm{V}$ acromio-clavicular Rockwood dislocations: clinical and radiological midterm results and MRI evaluation in 42 patients. Injury. 2012;43(2): 147-52.

10. Henkel T, Oetiker R, Hackenbruch W. Treatment of fresh Tossy III acromioclavicular joint dislocation by ligament suture and temporary fixation with the clavicular hooked plate. Swiss Surg. 1997;3(4):160-6.

11. Eschler A, Gradl G, Gierer P, Mittlmeier T, Beck M. Hook plate fixation for acromioclavicular joint separations restores coracoclavicular distance more accurately than PDS augmentation, however presents with a high rate of acromial osteolysis. Arch Orthop Trauma Surg. 2012;132(1):33-9.

12. Yoon JP, Lee YS, Song GS, Oh JH. Morphological analysis of acromion and hook plate for the fixation of acromioclavicular joint dislocation. Knee Surg Sports Traumatol Arthrosc. 2017; 25(3):980-6.

13. Fung $M$, Kato $S$, Barrance PJ, et al. Scapular and clavicular kinematics during humeral elevation: a study with cadavers. J Shoulder Elbow Surg. 2001;10(3):278-85.

14. Koukakis A, Manouras A, Apostolou CD, et al. Results using the $\mathrm{AO}$ hook plate for dislocations of the acromioclavicular joint. Expert Rev Med Devices. 2008;5(5):567-72.

15. Lin HY, Wong PK, Ho WP, Chuang TY, Liao YS, Wong CC. Clavicular hook plate may induce subacromial shoulder impingement and rotator cuff lesion: dynamic sonographic evaluation. J Orthop Surg Res. 2014;9:6.

16. Yoon JP, Chung SW, Kim JY, et al. Outcomes of Combined Bone Marrow Stimulation and Patch Augmentation for Massive Rotator Cuff Tears. Am J Sports Med. 2016;44(4):963-71.

17. Neviaser RJ, Neviaser JS, Neviaser TJ, Neviaser JS. A simple technique for internal fixation of the clavicle. A long term evaluation. Clin Orthop Relat Res. 1975;(109):103-7.

18. Kao FC, Chao EK, Chen CH, Yu SW, Chen CY, Yen CY. Treatment of distal clavicle fracture using Kirschner wires and tension-band wires. J Trauma. 2001;51(3):522-5.

19. Lisý M, Hreusík P, Steno B. The shape of the acromion and its effect on the subacromial space. Acta Chir Orthop Traumatol Cech. 2004;71(2):110-4.

20. Nyffeler RW, Werner CM, Sukthankar A, Schmid MR, Gerber C. Association of a large lateral extension of the acromion with rotator cuff tears. J Bone Joint Surg Am. 2006;88(4):800-5.

21. Haidar SG, Krishnan KM, Deshmukh SC. Hook plate fixation for type II fractures of the lateral end of the clavicle. J Shoulder Elbow Surg. 2006;15(4):419-23.

22. Meda PV, Machani B, Sinopidis C, Braithwaite I, Brownson 
P, Frostick SP. Clavicular hook plate for lateral end fractures: a prospective study. Injury. 2006;37(3):277-83.

23. Good DW, Lui DF, Leonard M, Morris S, McElwain JP. Clavicle hook plate fixation for displaced lateral-third clavicle fractures (Neer type II): a functional outcome study. J Shoulder Elbow Surg. 2012;21(8):1045-8.

24. Gstettner C, Tauber M, Hitzl W, Resch H. Rockwood type III acromioclavicular dislocation: surgical versus conservative treatment. J Shoulder Elbow Surg. 2008;17(2):220-5.

25. Ejam S, Lind T, Falkenberg B. Surgical treatment of acute and chronic acromioclavicular dislocation Tossy type III and V using the Hook plate. Acta Orthop Belg. 2008;74(4):441-5.
26. Jafary D, Keihan Shokouh H, Najd Mazhar F, Shariat Zadeh $\mathrm{H}$, Mochtary T. Clinical and radiological results of fixation of acromioclavicular joint dislocation by hook plates retained for more than five months. Trauma Mon. 2014;19(2):e13728.

27. Stegeman SA, Nacak H, Huvenaars KH, Stijnen T, Krijnen P, Schipper IB. Surgical treatment of Neer type-II fractures of the distal clavicle: a meta-analysis. Acta Orthop. 2013;84(2):18490.

28. Muramatsu K, Shigetomi M, Matsunaga T, Murata Y, Taguchi $\mathrm{T}$. Use of the AO hook-plate for treatment of unstable fractures of the distal clavicle. Arch Orthop Trauma Surg. 2007; 127(3):191-4. 